\title{
Hydrodynamic crystals: collective dynamics of regular arrays of spherical particles in a parallel-wall channel
}

\author{
M. Baron, J. Bławzdziewicz, and E. Wajnryl \\ Department of Mechanical Engineering, Yale University, P.O. Box 20-8286, New Haven, CT 06520
}

(Dated: October 22, 2018)

\begin{abstract}
Simulations of over $10^{3}$ hydrodynamically coupled solid spheres are performed to investigate collective motion of linear trains and regular square arrays of particles suspended in a fluid bounded by two parallel walls. Our novel accelerated Stokesian-dynamics algorithm relies on simplifications associated with the Hele-Shaw asymptotic far-field form of the flow scattered by the particles. The simulations reveal propagation of particle-displacement waves, deformation and rearrangements of a particle lattice, propagation of dislocation defects in ordered arrays, and long-lasting coexistence of ordered and disordered regions.
\end{abstract}

Long-range hydrodynamic interactions between solid particles suspended in a fluid result in complex collective dynamic phenomena, such as development of ordered arrays of magnetically driven rotors placed on a liquid interface 1] and formation of time-dependent patterns in a system of particles immersed in a vibrated fluid [2]. Collective behavior due to the hydrodynamic coupling also occurs in biological systems. A striking example is spontaneous formation of vortical arrays of self-propelled sperm cells confined to an interface [3]. Hydrodynamic coupling also plays an essential role in the synchronization of cilia beating and development of collective waves in cilia arrays in small swimming organisms [4].

In confined multiphase systems, the collective particle behavior is strongly influenced by bounding walls affecting the fluid motion. According to recent studies [5, 6, 6], hydrodynamic confinement effects are especially significant in parallel-wall channels of width comparable to the particle size. Lateral motion of a particle in such a channel produces fluid backflow that is involved in numerous dynamical phenomena. It enhances relative particle motion in confined quasi-2D-suspensions [5, 6], considerably increases transverse hydrodynamic resistance for elongated rigid arrays of spheres moving parallel to the channel walls [6], and governs propagation of particledisplacement waves [7] in linear arrays of drops in a microfluidic channel. We show that the fluid backflow resulting from particle motion is also responsible for pattern formation occurring in $2 \mathrm{D}$ hydrodynamic crystals (i.e. regular particle arrays that are hydrodynamically driven).

In this Letter we present a numerical study of the dynamics of $1 \mathrm{D}$ and $2 \mathrm{D}$ regular arrays of hydrodynamically coupled spherical particles in parallel-wall channels (cf. configurations shown in Fig. 1). We investigate propagation of displacement waves in linear arrays. In large square 2D arrays we report emergence of striking patterns, such as rearrangements of particle lattice, dislocation defects, and coexistence of ordered and disordered domains. We show that these patterns occur as a result of macroscopic deformation of a regular particle lattice, and we propose a macroscopic theory describing shape evolution of the arrays.

Our simulations are performed using a novel accelerated Stokesian-dynamics algorithm to follow evolution of about $10^{3}$ particles. Potential applications of our new algorithm include studies of collective motion of selfpropelled particles (e.g. bacterial colonies) in liquid films, modeling suspension flows in slit pores, and investigations of dynamics of macromolecules (e.g. DNA or polymer chains) in microfluidic channels. Our acceleration technique can also be used in boundary-integral algorithms for studying dynamics of deformable particles in confined geometry.

Our numerical technique relies on simplifications associated with the far-field asymptotics of the flow scattered from the particles. Far from a particle, the scattered flow in a parallel-wall channel assumes the Hele-Shaw form, i.e. it tends to a $2 \mathrm{D}$ parabolic flow that is driven by a harmonic pressure distribution [6]. In our new approach we expand the flow scattered by the particles into a carefully chosen fundamental set of Stokes flows. Close to a particle the basis flows form a complete set of solutions of Stokes equations in 3D space. In the far-field domain

(a)

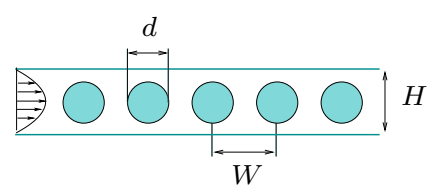

(b)

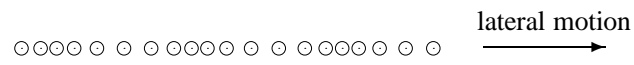

(c)

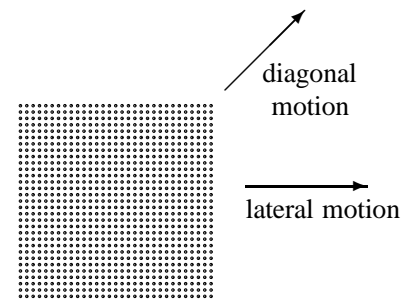

FIG. 1: Particle arrays in parallel-wall channels. (a) System definition; (b) lateral displacement wave in linear array; $(c)$ square array (top view). 


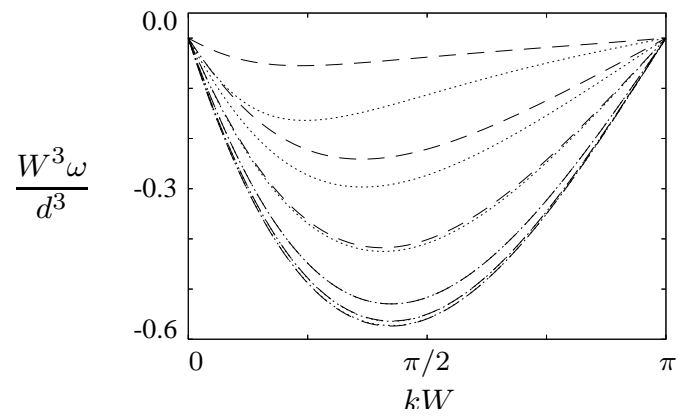

FIG. 2: Rescaled dispersion relation for small-amplitude longitudinal displacement waves in linear particle arrays in a channel of width $H=1.1 d$. Arrays driven by Poiseuille flow (dashed lines) and external force (dotted lines) for $W / d=$ $1.1,1.5,2,3,5,10$ (from above). The results for the forcedriven system are multiplied by the factor $\alpha=-0.325$.

$\rho \gg H$ (where $\rho$ is the lateral distance from the particle, and $H$ is the wall separation) these flows either exponentially tend to zero or to Hele-Shaw flow driven by a 2D pressure multipole. The expansion of the flow field into the new set of basis fields (obtained by an orthogonal transformation from the fields used in [6]) yields a sparse system of linear equations, which can be efficiently solved using iterative sparse-matrix-manipulation techniques. Moreover, since the far-field flow is uniquely determined by the harmonic pressure distribution, welldeveloped acceleration techniques for Laplace equation can be applied to further increase numerical efficiency. The simulations discussed below show that our algorithm is efficient and highly accurate in both the far-field and the near-field regimes. The calculations also reveal surprisingly rich collective particle dynamics.

Figures 2 and 3 present our results for propagation of particle displacement waves in an infinite train of equally spaced particles positioned along a line in the midplane of a channel slightly wider than the particle size. The particle array is driven either by Poiseuille flow [cf. Fig. [1(a)] or by a constant external lateral force. We focus on the longitudinal waves, where the particle displacements $\delta x_{i}$ from the reference positions $x_{i}=i W(i=0,1,2 \ldots)$ on a regular lattice with spacing $W$ occur along the array [cf. Fig. 1 $1(b)]$.

Figure 2 shows the dispersion relation $\omega=\omega(k)$ for harmonic displacement waves $\delta x_{i}=\epsilon \sin \left(k x_{i}-\omega t\right)$ in arrays with different interparticle spacing. Here $k$ is the wave number, $\omega$ is the wave frequency measured in the reference frame moving with the particles, and $\epsilon \ll 1$ is the wave amplitude. The time and frequency are normalized by the time $\tau_{0}$ in which an isolated particle in a channel moves by the diameter $d$. The shape of the dispersion curves is reflected in the evolution of wave packets depicted in Fig. 3, For small interparticle spacing the maximum frequency is shifted towards smaller wave vectors, because the lubrication forces hinder the relative
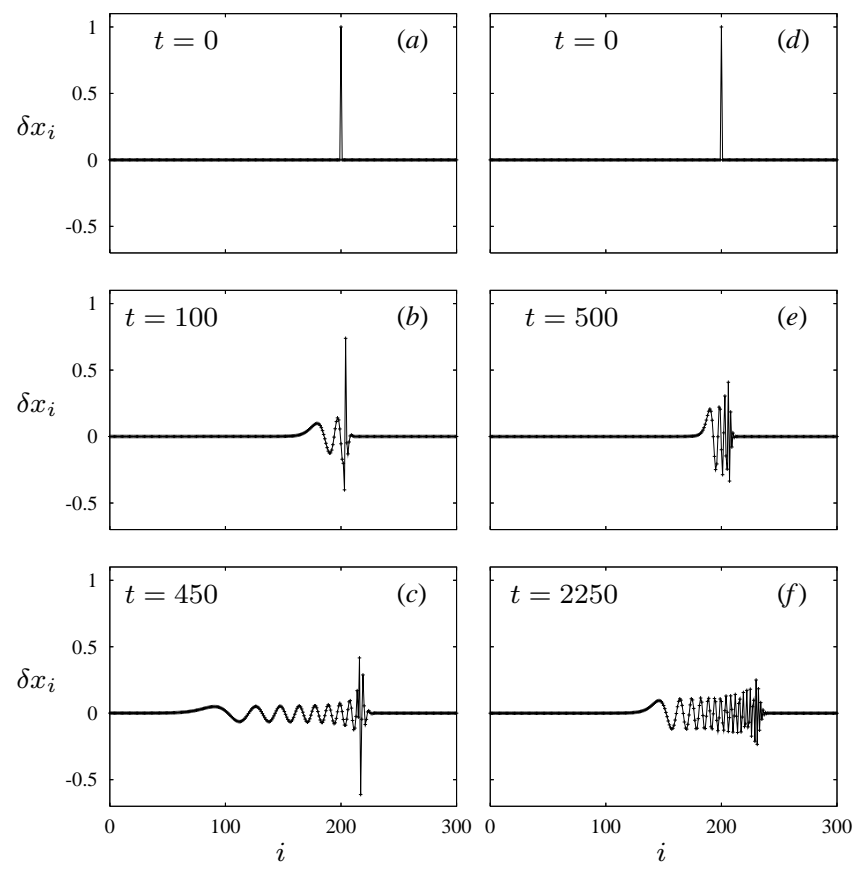

FIG. 3: Evolution of a wave packet in an array with particle spacing $W / d=1.1(a-c)$ and $W / d=3(d-f)$. Particle displacements $\delta x_{i}(i=0,1, \ldots)$ are normalized by the magnitude of the initial perturbation.

particle motion. Hence, there is a long-wave tail in the wave packet shown in Fig. 3) $(c)$.

In Fig. 2 the frequency $\omega$ is plotted rescaled by a factor $(W / d)^{3}$ to emphasize the universal behavior of the system for large values of $W$. In addition, the results for the force-driven train are multiplied by a constant negative factor $\alpha$. We find that for $W / d \gtrsim 5$ all rescaled results fall onto a single asymptotic master curve. In the regime $W / d \approx 2-3$ the dispersion relations significantly deviate from the master curve, but the scaled results for the flowand force-driven arrays are still nearly identical. Since the scale factor $\alpha$ is negative, this behavior indicates that for moderate and large interparticle distances the relative particle motion in an array driven by an external flow is equivalent to the relative motion in a force-driven array moving in the opposite direction.

The above features of the system dynamics result from the far-field behavior of the flow field scattered by the particles. In the far-field regime an isolated particle subject to Poiseuille flow or external force produces the same Hele-Shaw flow $\mathbf{v}^{\mathrm{HS}}$ driven by the two-dimensional dipolar pressure $p^{\mathrm{HS}} \sim \cos \phi / \rho$ (where $\phi$ is the polar angle measured from the direction of the external forcing) [5, [6]. For $W / d \gtrsim 5$ the single-scattering approximation corresponding to the superposition of dipolar fields $\mathbf{v}^{\mathrm{HS}}$ adequately describes the system dynamics (and thus the rescaled results in Fig. 2 follow a single master curve). In the regime $W / d \approx 2-3$ evaluation of a single flow reflection is insufficient; nevertheless, the results for flow- and 
(a)

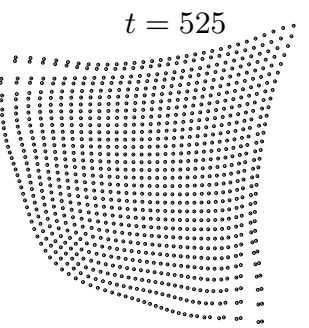

(b)

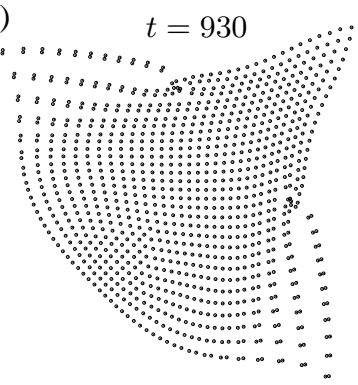

(c)

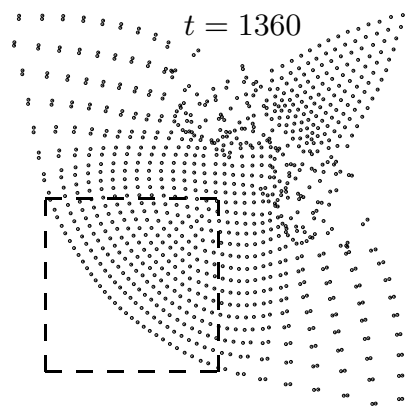

(d)

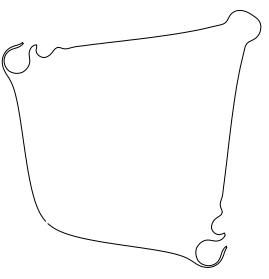

(e) $\quad t=525$

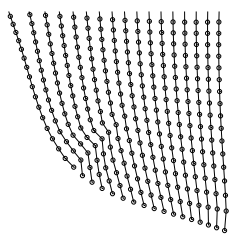

$(f)$

$t=930$

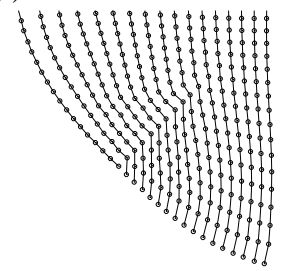

$(g)$

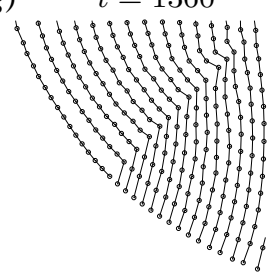

FIG. 4: Evolution of a force-driven square array of $N=961$ particles, moving in the diagonal direction. Channel width $H / d=1.1$ and initial particle spacing $W / d=5$ (particles are shown magnified by a factor of 2). (a-c) Simulation results; $(d)$ prediction of mean-field theory for the same stage of evolution as in $(a) ;(e-g)$ development of a fault line in the indicated region.

force-driven arrays can be rescaled onto each other. This is because the first reflection in the multiple-scattering sequence for the two systems is nearly identical (apart from a rescaling factor), owing to the exponential approach of the flow field to the asymptotic Hele-Shaw form $\mathbf{v}^{\mathrm{HS}}$. With matching initial reflections, the whole multiple-scattering sequences for systems with different forcing coincide, and the relative particle motion is thus the same. This argument is valid not only for linear arrays but also for other horizontal particle arrangements (such as the 2D arrays shown in Figs. 46). Moreover, similar reasoning applies to different kinds of forcing, including Marangoni and electrophoretic forces used to control particle positions in microfluidic devices.

Rich collective phenomena revealed by our simulations of 2D hydrodynamic crystals are illustrated in Figs. 46. Figure 4 presents the evolution of a regular square

array [cf. Fig. 1( $(c)$ ] of about $10^{3}$ particles undergoing diagonal motion produced by a constant force acting on all the particles. The initial particle spacing is within the far-field asymptotic regime, $W / d=5$. Figure 5 shows corresponding results for lateral motion of the array.

Our simulations demonstrate that at short times a deforming square array retains its initial particle ordering [cf. Fig. 4( $(a)]$. Subsequently, the system develops some striking structural features. Several rows of particle pairs separate from the main body of the array, forming a shape similar to airplane wings. The front part of the array has an approximately hexagonal particle ordering, and the middle part retains the square ordering. The rear part [marked region in Fig. 4( $(c)$ ] has a square particle arrangement but with a different orientation than the original one. The blowup in Fig. $4(e-g)$ shows that the particle rearrangement involves discontinuous particle displacements along a "fault line" at the symmetry axis of the array. A similar dislocation event (but without lattice reorientation) is observed in an array in the lateral motion (cf. Fig. 5). There also occur instabilities responsible for emergence of disordered domains of chaotic particle motion. In the diagonal motion [Fig. [4 $(b, c)]$ the instability starts at the junction between the wings and the body of the array. For the lateral motion, (a)
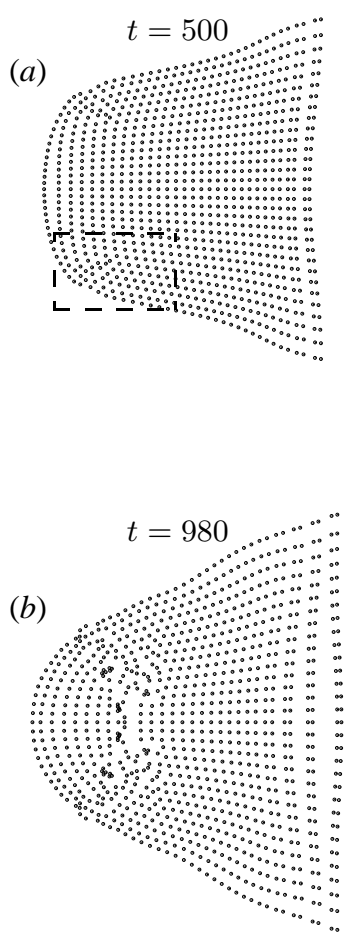

(c)

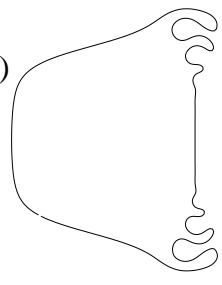

(d)

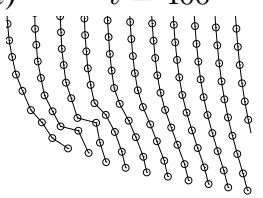

(e)

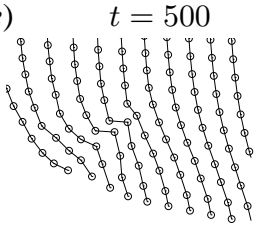

(f)

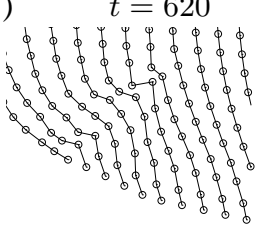

FIG. 5: Same as Fig. 4 but for lateral motion of the array. $(a, b)$ Simulation results; $(c)$ prediction of mean-field theory for the same stage of evolution as in $(a) ;(d-f)$ development of a dislocation line in the indicated region. 

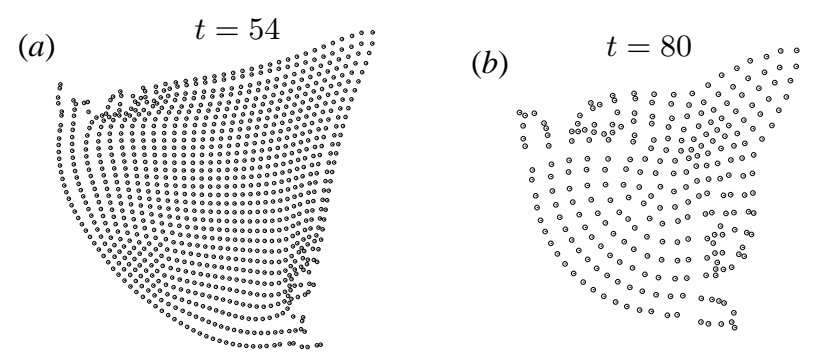

FIG. 6: Square arrays of $(a) N=961$ and (b) $N=256$ particles in diagonal motion. Channel width $H / d=1.1$ and initial particle spacing $W / d=2$.

the order-disorder transition occurs when the dislocation lines approach the center of the array [Fig. [5 (b)].

Figures 4 and 5 demonstrate that the ordered regions can withstand large macroscopic deformations and random perturbations originating from the disordered domains. Moreover, the ordered crystalline domains can rearrange and heal themselves along fault or dislocation lines. The strong propensity to maintain the ordered structure results from the dipolar hydrodynamic interactions of neighboring particles. An array also undergoes a macroscopic deformation resulting from the combined long-range effect of the dipolar flow fields produced by individual particles.

For low-density arrays (i.e. for $W / d \gg 1$ ) the macroscopic flow that causes the deformation can be determined from the flow field produced by a uniform distribution of pressure dipoles $p^{\mathrm{HS}}$ induced in the array. In general, the macroscopic deformation can be described using the effective 2D transport equations for suspension flow in a parallel-wall channel, $\mathbf{u}=-\nu_{p} \boldsymbol{\nabla} \bar{p}+\nu_{f} \mathbf{f}$, and $\mathbf{j}_{p}=-\mu_{p} \boldsymbol{\nabla} \bar{p}+\mu_{f} \mathbf{f}$. Here $\mathbf{u}$ is the suspension velocity averaged across the channel, $\mathbf{j}_{p}$ is the particle flux, $\mathbf{f}$ denotes the density of the lateral force acting on the particles, $\bar{p}$ is the macroscopic pressure, and $\nu_{\alpha}, \mu_{\alpha}(\alpha=p, f)$ are linear transport coefficients. The suspension velocity and suspension flux satisfy the continuity equations $\boldsymbol{\nabla} \cdot \mathbf{u}=0$ and $\partial \bar{\rho} / \partial t=-\boldsymbol{\nabla} \cdot \mathbf{j}_{p}$, where $\bar{\rho}$ is the suspension density.

The macroscopic deformation of an array, evaluated in the uniform-dipolar-moment approximation, is shown in Figs. $4(d)$ and $5(c)$. The results indicate that our macroscopic description reproduces the overall shape of the arrays for moderate times (i.e. before the complex structural features develop).

We note that the macroscopic equations predict fingering instabilities near the array corners. In low-density arrays (cf. Figs. 4and 5) such instabilities are suppressed due to the array "stiffness" associated with its ordered structure. However, for denser arrays (cf. Fig. 6) the macroscopic deforming forces are sufficiently strong to destabilize the tips of the array wings, in agreement with our macroscopic theory. The results in Fig. 6 indicate that the lengthscale for the fingering instability in par- ticle arrays is determined by the particle lattice. In our effective medium theory there is no intrinsic lengthscale, so the size of the fingers in Figs. $4(d)$ and $5(c)$ is set by the initial condition (i.e. a square with rounded corners).

The patterns we observe in 2D hydrodynamic crystals have analogies in other athermal systems. For example, dislocations and chaotic dynamics develop in arrays of convective cells in a fluid undergoing Benard convection [8] and in vibrated granular media [9]. Our system has a number of interesting distinctive features. First, the pattern formation occurs in the linear Stokesflow regime, and the nonlinearity stems entirely from the position-dependence of the multiparticle mobility matrix. Next, the dipolar hydrodynamic interactions that maintain particle ordering are non-isotropic (causing, e.g., lattice reorientation). Finally, the dipolar flow $\mathbf{v}^{\mathrm{HS}}$ not only maintains the ordered structure on the local level but also produces the macroscopic deformation of the array, leading to lattice instabilities.

Regular particle arrays can be assembled using holographic optical tweezers [10], so the collective dynamic phenomena revealed by our study should be accessible experimentally. The effect of hydrodynamic coupling on the motion of regular particle arrays could also be observed in flow-driven 2D colloidal crystals. The equivalence of the relative particle motion in systems with different forcing can be used to separately control the relative particle positions and the position of the center of mass of an array.

This work was supported by NSF CAREER grant CBET-0348175 and MNiSW grant N501 020 32/1994.

* On leave from IPPT Warsaw, Poland.

[1] B. A. Grzybowski, H. A. Stone, and G. M. Whitesides, Nature 405, 1033 (2000); P. Lenz et al., Phys. Rev. Lett. 91, 108104 (2003).

[2] G. A. Voth et al., Phys. Rev. Lett. 88, 234301 (2002).

[3] I. H. Riedel, K. Kruse, and J. Howard, Science 309, 300 (2005).

[4] P. Lenz and A. Ryskin, Phys. Biol. 3, 285 (2006); A. Vilfan and F. Julicher, Phys. Rev. Lett. 96, 058102 (2006).

[5] B. Cui, H. Diamant, B. Lin, and S. A. Rice, Phys. Rev. Lett. 92, 258301 (2004).

[6] S. Bhattacharya, J. Bławzdziewicz, and E. Wajnryb, J. Fluid Mech. 541, 263 (2005); J. Comput. Phys. 212, 718 (2006).

[7] T. Beatus, T. Tlusty, and R. Bar-Ziv, Nature Phys. 2, 743 (2006); Phys. Rev. Lett. 99, 124502 (2007).

[8] Y.-N. Young and H. Riecke, Phys. Rev. Lett. 90, 134502 (2003).

[9] I. S. Aranson and L. S. Tsimring, Rev. Mod. Phys. 78, 641 (2006).

[10] M. Polin, D. G. Grier, and S. R. Quake, Phys. Rev. Lett. 96, 088101 (2006); S. C. Chapin, V. Germain, and E. R. Dufresne, Opt. Express 14, 13095 (2006). 